\title{
Improve the Government Strategic Petroleum Reserves
}

\author{
Xiucheng Dong, Zhongbing Zhou", Hui Li \\ China Oil \& Gas Centre, Beijing, China \\ Email: *bennychow09@163.com
}

Received June 21, 2013; revised July 21, 2013; accepted July 31, 2013

Copyright (C) 2013 Xiucheng Dong et al. This is an open access article distributed under the Creative Commons Attribution License, which permits unrestricted use, distribution, and reproduction in any medium, provided the original work is properly cited.

\begin{abstract}
The potentiality that the current government strategic petroleum reserves (GSPRs) can be improved by the pre-allocation of GSPR drawing rights has been neglected. This paper proposes to pre-allocate the GSPR drawing rights, and proves that by doing this the efficiency of GSPR and the society's incentive to finance GSPR can be improved. Particularly, the example demonstrates that the incentive improvement can be very significant. Since it takes huge expenditure on GSPR and it is very important to gain support from the consumers by improving GSPR, the proposal is quite worth considering.
\end{abstract}

Keywords: Strategic Petroleum Reserve; Panic Buying; Drawing Rights Allocation; Efficiency

\section{Introduction}

To prepare for oil supply interruption in advance, strategic petroleum reserves (SPRs) [1] across the countries with high dependency on imported oil have been built. Almost $1 / 3$ of SPRs are government owned stocks [2], or what we call government strategic petroleum reserves (GSPRs). GSPR is vulnerable to criticisms and the society may have weak incentives to support it, because it is totally financed by public expenditure. The institution CATO published an analysis in 2005, arguing that the US SPR programs were inefficient since they have cost the US citizens too much but only generated a little benefit [3]. The criticisms on GSPRs motivated us to come up with a proposal for improving the GSPR. Based on strong assumptions, the proposal reserves a great possibility to improve the current GSPRs.

The assumptions and the proposal will be elaborated in Section 2. Section 3 shows that how the proposal is supposed to improve the current GSPR in terms of efficiency, and Section 4 proves that the proposal can increase the society's incentive to support GSPRs. The significance and limitedness of the proposal will be discussed in Section 5 . Section 6 concludes the paper.

\section{The Assumptions and the Proposal}

\subsection{The Assumptions}

SPRs generate two kinds of good. First, SPRs can keep

\footnotetext{
"Corresponding author.
}

oil price from soaring at supply interruption; second, SPR drawing rights enable the owners to be more competitive for the released SPR oil and thus give them advantages at supply interruption. The first good benefits all oil consumers and excludes none from enjoying it at bearable cost, so for it we have the name "public good", which is typically a kind of Samuelson's pure public goods [4]. The second good can easily exclude any one by price biding, so for it we have the name "private good".

Two government types are defined as

$G_{0}\left(I, M, U_{0}, C, t, r\right)$ and $G_{1}\left(I, M, U_{0}+U_{1}, C, t, r\right)$, where $I$ is the number of the oil consumers, $M$ is the size of the GSPR the government need build, $U_{0}$ is the aggregate utility function of GSPR as pure public good while $U_{1}$ is the aggregate utility function of the pre allocated GSPR drawing right, $C$ is the GSPR's total cost, $t$ is the anticipated duration between the time 0 and the next supply interruption, and $r$ is the real rate incorporating factors of the oil price's long run trend. The difference between the two governments is that under $G_{0}$ GSPR is purely financed by public expenditure, while under $G_{1}$ GSPR is financed not only by public expenditure but also by the private bids for the GSPR drawing rights before the construction of GSPR. For the governments, assumptions 1-3 are arranged.

Assumption 1: Both $G_{0}$ and $G_{1}$ know the function $C(M)$, which is concave and continuously increasing on $M$.

Assumption 2: $G_{0}$ knows $U_{0}$, and $G_{1}$ knows both 
$U_{0}$ and $U_{1}$.

Assumption 3: As net oil importer, both $G_{0}$ and $G_{1}$ maximizes the aggregate consumer surplus, which means $\partial U_{0} / \partial M=\partial C / \partial M$ must hold for $G_{0}$, and $\partial U_{0} / \partial M+\partial U_{1} / \partial M=\partial C / \partial M$ must hold for $G_{1}$.

Consumers are willing to pay for GSPR because they have expected an oil supply interruption which may endanger their welfare and this danger may cost them more than GSPR does. As price takers under the current oil market condition, oil consumers know well that only collective actions can deter the soaring oil price. Economies of scale provide a main rationale for considering public infrastructure provision [5]. GSPR projects have extraordinary economies of scale, and the total GSPR cost would be unacceptably high if construction actions have been taken separately rather than collectively.

Let $u_{i}(M)$ stand for consumer $i$ 's utility function of GSPR as public good, and $u_{i \mathrm{~d}}\left(\lambda_{i}\right)$ stand for consumer i's utility of his/her GSPR drawing right $\lambda_{i}, C_{i}$ stand for consumer i's payment for GSPR, $B_{i}$ stand for i's proportional share of the total cost pie of the GSPR, and $\sum \lambda_{i}=M$. For the consumers, assumptions 4-6 are arranged.

Assumption 4: $u_{i}$ is concave and continuously increasing on $M ; u_{i \mathrm{~d}}$ is concave and continuously increasing on $\lambda_{i} ; \sum u_{i}=U_{0}$, and $\sum u_{i \mathrm{~d}}=U_{1}$.

Assumption 5: $\partial u_{i} / \partial M+\partial u_{i} / \partial \lambda_{i}=\partial C_{i} ; C_{i}=B_{i} c(M)$, where $0 \leq B_{i} \leq 1$ and $\sum B_{i}=1$.

Assumption 6: There exists certain $\underline{M}$ so that in $[0, \underline{M}], C \leq U_{0}+U_{1}$ while in $(\underline{M},+\infty), C>U_{0}+U_{1}$.

It can be inferred that $U=\sum\left(u_{i}+u_{i d}\right)$ is also continuously increasing on $M$. However, there is free-riding problem in the provision of public good and it's rather difficult to overcome this problem by decentralized mechanisms, so for simplicity we assume that under the government' authority, the society's propensity to free-ride has been wiped out. Under this circumstance, the government can optimally decide the size of the GSPR as public good and the distribution of the corresponding cost. The rationale of the last sentence of assumption 6 is, if $C$ is always larger than $U_{0}+U_{1}$, GSPR should never be built; and, that $U_{0}+U_{1}$ is always larger than $C$ is impossible.

We introduce the multiplier $k(0<k \leq 1)$ to further clarify the difference between the concepts of GSPR as public good and that of the GSPR drawing right. If there exists and only exists one $k$ so that the cost of the GSPR as public good is $k C(M)$ and that of the GSPR drawing right cost is $(1-k) C(M)$, the GSPR as public good and the GSPR drawing right are clearly separated. Under this circumstance, $B_{i}$ becomes the coordinate of i's share of the public good cost and that of the drawing right cost, $\left(\beta_{i}, b_{i}\right)$, subject to $\sum \beta_{i}=1$ and $\sum b_{i}=1$.

Many examples, such as panic buying of fuel in 1973 oil crisis [6], panic buying of fuel in Hurricane Katrina [7], panic buying of salt in Japan' s nuclear crisis [8] and so on, suggest that consumer hoarding (or panic buying) may happen at supply interruption. According to the economic explanation given by [9], panic buying can be interpreted as the distortion of demand curve. When supply interruption happens, demanders are expected to value the supply unusually high and get less elastic to price, hence the demanders may suffer greater surplus loss. For the possibility of panic buying, assumption 7 is arranged.

Assumption 7: The announced supply interruption distorts the price elasticity of the aggregate demand. The degree of the distortion is decreasing on the size of the strategic inventory held at the immediate convenience of the consumers.

\subsection{The Proposal}

Suppose that the oil supply interruption is expected to take pace at time $t$ and policies of GSPR are required to make at time 0 . Currently, no GSPR drawing right has been pre allocated and the GSPR oil is sold to the market at $P_{t}$, the instant market price of time $t$, which means at time $0, k$ is regarded as 1 . In other words, the current government is typically the kind of $G_{0}$. Our proposal is requiring the government to transform from $G_{0}$ to $G_{1}$.

More specifically, consumers and $G_{1}$ at time 0 are proposed to sign a contract which specifies: at time $t$ consumer $i$ is allowed to buy $\lambda_{i}$ amount of GSPR stock at the price $P_{0} e^{r t}$, where $P_{0}$ is the market price at the contracting time point, provided that at time 0 consumer $i$ pays a fair part of the total GSPR cost. To differentiate the GSPR under $G_{1}$ from the normal commercial oil stocks, we emphasize that the drawing rights also can only be executed at the government announced oil supply interruption.

\section{The Proof of the Efficiency Improvement}

Suppose at supply interruption, the oil supply is suddenly reduced to $S$ from the normal level $S$, and the size of the GSPR is $M$.We can do a geometric analysis and see there is a potential improvement between the ex ante and the ex post situations. In Figure 1, the abscissa stands for the amount of oil the consumers buy and the ordinate stand for the oil price; $\alpha$ marks point $\left(q_{2}, g\right), b$ marks point $\left(q_{1}, h\right), c$ marks point $(q, l), d$ marks point $\left(q_{1}, m\right)$, and $e$ marks point $\left(q_{2}, n\right)$.

In ex ante situation, the demanders have a total quantity $M$ of GSPR drawing rights in hands and they can use it once the government declares a severe oil supply interruption. In this situation, the aggregate inverse demand function is distorted to $P_{1}(Q)$, and the consumers buy oil of amount $q_{1}$ need pay price $l$ averagely. But, at 


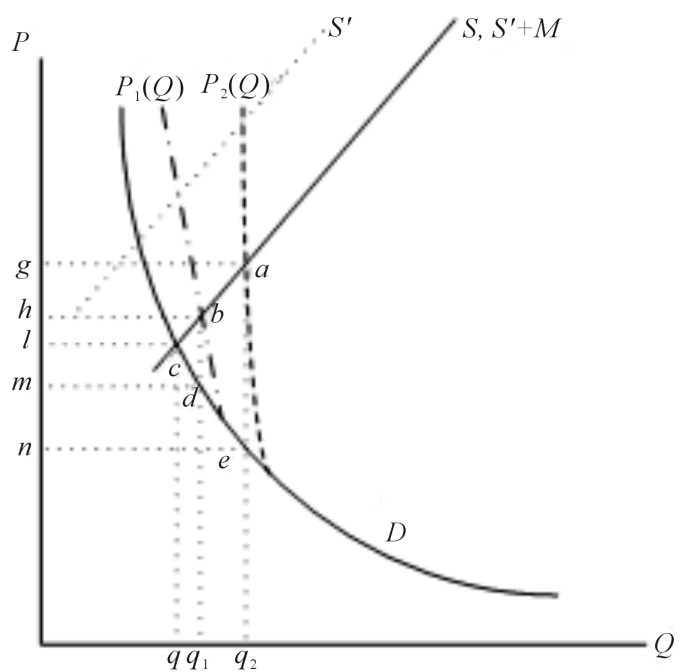

Figure 1. Ex ante preparation improves the GSPR by relieving panic buying.

normal state the inverse demand function is $P_{1}(Q)$ (or $D$ in Figure 1) and the consumers only need to pay the average price $e$ for the same amount of oil. Thus, the consumers' surplus loss of is the size of trapezoid hbcl plus the size of curved triangle bcd, or $(h-m) q_{1}-\int_{g}^{q_{1}} D d Q$.

In ex post situation, the demanders have little immediate strategic stockpile. In this situation, a round of chaotic panic buying would happen, and the aggregate inverse demand function would be distorted to $P_{2}(Q)$. The consumer surplus loss in ex post situation is measured by the size of trapezoid gacl plus the size of curved triangle ace, or $(g-n) q_{2}-\int_{q}^{q_{2}} D d Q$.

Apparently, $(h-m) q_{1}-\int_{q}^{q_{1}} D d Q$ is always less than $(g-n) q_{2}-\int_{q}^{q_{2}} D d Q$, hence the ex ante preparation is better than the ex post one in terms of efficiency.

\section{The Proof of the Incentive Improvement}

\subsection{The General Proof}

By incentive improvement we meant that under the proposed mechanism, consumers will enable the government to build a lager GSPR by contributing more. Under $G_{1}$, there exists one and only one $k(0<k<1)$ so that $\partial U_{0} / \partial M=k \partial C / \partial M$ and $\partial U_{1} / \partial M=(1-k) \partial C / \partial M$. Given the GSPR of size $M$, all consumers will pay less for GSPR as public good for $k C(M)$ is definitely less than $C(M)$. Therefore, the aggregate marginal utility curve under $G_{1}$ intersects the marginal cost curve to the right (on the vertical line $M=M_{1}$ ) of the point where the marginal utility curve under $G_{0}$ intersects the marginal cost (on the vertical line $M=M_{0}$ ), as Figure 2 shows. Thus, the government $G_{1}$ is required to build a GSPR of size $M_{1}$, and the total expenditure is $C\left(M_{1}\right)$ which is obviously larger than $C\left(M_{0}\right)$. Thus, the incen- tive improvement has easily been proved.

From Figure 2, it can also be easily inferred that the significance of the incentive improvement depends on the scale of $k$ : the smaller the $k$ the larger the $M_{1}$, which means if the consumers value GSPR drawing rights more, the incentive improvement would be more significant.

\subsection{An Example}

In order to deepen the understanding of the incentive improvement, we proceed with a simple numerical example.

Suppose $C(M)=6 M-1 /(M+1)+1, \quad I=3$, and respectively the consumers' utility functions are:

$$
\begin{aligned}
& u_{1}=\ln (M+1)-1 /(M+1)+1 \\
& u_{1 \mathrm{~d}}\left(\lambda_{1}\right)=2 \ln (\lambda+1)-1 /(\lambda+1) \\
& u_{2}=2 \ln (M+1)-2 /(M+1)+2 \\
& u_{2 \mathrm{~d}}\left(\lambda_{2}\right)=3 \ln \left(\lambda_{2}+1\right)-1 /\left(\lambda_{2}+1\right)+1 \\
& u_{3}=3 \ln (M+1)-3 /(M+1) \\
& u_{3 \mathrm{~d}}\left(\lambda_{3}\right)=4 \ln \left(\lambda_{3}+1\right)-1 /\left(\lambda_{3}+1\right)+1
\end{aligned} .
$$

It can be inferred that, under this market the aggregate utility function of the GSPR as public good is $U_{0}(M)=6 \ln (M+1)-6 /(M+1)+6$, and that of the GSPR drawing right is

$$
U_{1}(M)=9 \ln (M / 3+1)-3 /(M / 3+1)+3 .
$$

Under $G_{0}, k=1$ and the optimal $M_{0}$ is determined by Equation (1) according to the principle "marginal utility equals marginal cost”.

$$
5 /\left(M_{0}+1\right)^{2}+6\left(M_{0}+1\right)-6=0
$$

and, the cost distribution among the consumers is determined by Equation (2).

$$
\left\{\begin{array}{l}
5 /\left(M_{0}+1\right)^{2}+6\left(M_{0}+1\right)-6=0 \\
1 /\left(M_{0}+1\right)+1 /\left(M_{0}+1\right)^{2}=\beta_{1}\left[6+1 /\left(M_{0}+1\right)^{2}\right] \\
2 /\left(M_{0}+1\right)+2 /\left(M_{0}+1\right)^{2}=\beta_{2}\left[6+1 /\left(M_{0}+1\right)^{2}\right] \\
3 /\left(M_{0}+1\right)+3 /\left(M_{0}+1\right)^{2}=\beta_{3}\left[6+1 /\left(M_{0}+1\right)^{2}\right] \\
\beta_{1}+\beta_{2}+\beta_{3}=1
\end{array}\right.
$$

By solving the combination of Equation (1) and Equation (2), we can obtain that, under $G_{0}, M_{0}=0.54$, the total expenditure on the GSPR is 3.60 , and the cost distribution is $\beta\left(\beta_{1}, \beta_{2}, \beta_{3}\right)=(0.167,0.33,0.5)$ in terms of proportions, or the consumers need pay $0.60,1.20$ and 1.80 respectively.

Under $G_{1}, 0<k<1$ and the optimal $M_{1}$ is determined by Equation (3). 


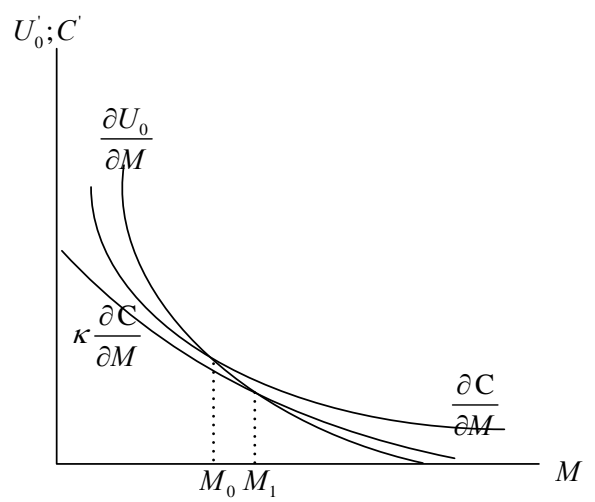

Figure 2. The intersections of the aggregate marginal utility curve and the marginal cost curves under $G_{0}$ and $G_{1}$.

$$
\frac{6}{M_{1}+1}+\frac{6}{\left(M_{1}+1\right)^{2}}+\frac{9}{M_{1}+3}+\frac{9}{\left(M_{1}+3\right)^{2}}=6+\frac{1}{\left(M_{1}+1\right)^{2}}
$$

and, the cost distribution among the consumers is determined by Equation (4).

$$
\left\{\begin{array}{l}
1 /\left(M_{1}+1\right)+1 /\left(M_{1}+1\right)^{2}=\beta_{1} k\left[6+1 /\left(M_{1}+1\right)^{2}\right] \\
2 /\left(M_{1}+1\right)+2 /\left(M_{1}+1\right)^{2}=\beta_{2} k\left[6+1 /\left(M_{1}+1\right)^{2}\right] \\
3 /\left(M_{1}+1\right)+3 /\left(M_{1}+1\right)^{2}=\beta_{3} k\left[6+1 /\left(M_{1}+1\right)^{2}\right] \\
2 /\left(\lambda_{1}+1\right)+1 /\left(\lambda_{1}+1\right)^{2}=b_{1}(1-k)\left[6+1 /\left(M_{1}+1\right)^{2}\right] \\
3 /\left(\lambda_{1}+1\right)+1 /\left(\lambda_{1}+1\right)^{2}=b_{2}(1-k)\left[6+1 /\left(M_{1}+1\right)^{2}\right] \\
4 /\left(\lambda_{1}+1\right)+1 /\left(\lambda_{1}+1\right)^{2}=b_{3}(1-k)\left[6+1 /\left(M_{1}+1\right)^{2}\right] \\
\beta_{1}+\beta_{2}+\beta_{3}=M_{1} \\
b_{1}+b_{2}+b_{3}=1
\end{array}\right.
$$

By solving the combination of Equation (3) and Equation (4), we can obtain that, under $G_{1}, M_{1}=1.351$, $k=0.55$, the total expenditure on the GSPR as public good is 4.81 and the total expenditure on the GSPR drawing rights is 3.86 , and the cost distribution of the GSPR as public good is $(0.167,0.33,0.5)$ in terms of proportions or $(0.80,1.60,2.41)$ in terms of monetary values; the cost distribution of the GSPR drawing rights is $(0.175,0.337,0.488)$ in terms of proportions or $(0.68,1.30,1.89)$ in terms of monetary values; and, $\left(\lambda_{1}, \lambda_{2}, \lambda_{3}\right)=(0.236 .0 .455,0.660)$. We can see that there has been a very significant improvement since $M_{1}$ is more the twice of $M_{0}$ and all together the consumers will spend more income under $G_{1}$ then the twice of that under $G_{0}$.

\section{Discussion}

Sections 3 and 4 have theoretically proved that the pro- posal is supposed to improve the current GSPR, this improvement has practical difficulty though. To be optimal, balanced and impartial, the government $G_{1}$ shall make sure that, given $M_{1}, \sum \beta_{i}=1$ on one side, and $\sum \lambda_{i}=M_{1}$ on the other. Those equalities require that each consumer reports to $G_{1}$ his/her utilities functions honestly. We have assumed that under both $G_{0}$ and $G_{1}$, the consumers give up their propensity to free-ride on others. This assumption doesn't reflect the possibility that $G_{1}$ would reinforce the consumers' propensity to free-ride, since some consumers may think the others have stronger desire for the GSPR drawing rights, and even they choose to pay nothing a GSPR will be financed. However, it's really more difficult to solve the free-riding problem under $G_{0}$ than $G_{1}$ ? This question needs in-depth investigation into the consumers' behavioral motives and the legitimacy of the governmental authority to answer, which transcends the scope of this paper. Nevertheless, the proposal reserves a great possibility to improve the current GSRP as the example suggests, hence still worth serious considering.

\section{Conclusion}

GSPR programs are supposed to save the probable larger loss with a confirmed cost, so the efficiency of them is very important. For historical events have suggested that panic buying is quite likely to happen at supply interruptions, we argue that any in-advance preparation for emergencies should have better immediacy. GSPR can be more immediate if its drawing rights have been pre-allocated. Moreover, this pre-allocation may greatly improve the society's incentive to finance GSPR. Therefore, we propose that when deciding the size and the cost distribution of GSPR at time 0, the GSPR drawing rights shall be allocated simultaneously. The GSPR drawing rights specify that if an oil supply interruption happens at time $t$, consumer $i$ who has bought the GSPR drawing right of $\lambda_{i}$ is allowed to buy the released GSPR oil of amount $\lambda_{i}$ at the preset price $P_{0} e^{r t}$, where $P_{0}$ is the market price at time 0 . Of course, the GSPR drawing rights can only be executed at the government that declares oil supply interruption.

\section{Acknowledgements}

This paper was founded by the Ministry of Education of the People's Republic of China under the name of its Philosophy and Social Science Major Issue Research Project "Research on Expanding the Oil \& Gas Strategic Reserve" (Grant No. 11JZD048) and National Natural Science Foundation of China under the name of its project "Research on the System of Natural Gas Security \& Emergency Measures" (Grant No. 71273277). We owe the greatest thanks to those two founders. However, we 
have to say at all this paper doesn't necessarily represent the opinion of those two founders.

\section{REFERENCES}

[1] Z. M. Liu, L. W. Zhu and J. M. Wang, "Optimization of China's Strategic Petroleum Reserve Policy: A Markovian Decision Approach," Computers \& Industrial Engineering, Vol. 63, No. 3, 2012, pp. 626-633. http://dx.doi.org/10.1016/j.cie.2011.10.014

[2] International Energy Agency, "IEA Response System for Oil Supply Emergencies,” 2012 Edition, 2012.

http://www.iea.org/publications/freepublications/publicati on/name,3714,en.html

[3] J. Taylor and P. V. Doren, "The Case against the Strategic Petroleum Reserve,” Cato Institute, Washington DC, 2005.

[4] P. A. Samuelson, "The Pure Theory of Public Expenditure," The Review of Economics and Statistics, Vol. 36, No. 4, 1954, pp. 387-389. http://dx.doi.org/10.2307/1925895

[5] D. Holtz-Eakin and M. E. Lovely, "Scale Economies, Returns to Variety, and the Productivity of Public Infra- structure," Regional Science and Urban Economics, Vol. 26, No. 2, 1996, pp. 105-123.

http://dx.doi.org/10.1016/0166-0462(95)02126-4

[6] A. S. Cooper, "A Tale of Two Oil Shocks. Part 1: 197376,” Frontline, 2012.

http://www.pbs.org/wgbh/pages/frontline/tehranbureau/2 012/06/a-tale-of-two-oil-shocks-part-1-1973-

[7] E. Chow and J. Elkind, "Hurricane Katrina and US Energy Security,” Survival, Vol. 47, No. 4, 2005, pp. 145160. http://dx.doi.org/10.1080/00396330500433449

[8] J. C. Wei, L. Zhou and X. Zhou, "Analysis of the Evolution Mechanism of Mass Panic Buying under the Public Crisis. Case Study on Salt Panic Buying in China during the Japan Nuclear Crisis," Journal of Management Case Studies, Vol. 4, No. 6, 2011, pp. 478-486.

[9] S. Ronald, J. Keith and A. T. Khairy, "Scarcity and Hoarding: Economic and Social Explanations and Marketing Implications," Advances in Consumer Research, Vol. 2, 1975, pp. 203-216. 